Bundesgesundheitsbl $2021 \cdot 64: 384$

https://doi.org/10.1007/s00103-021-03286-0

Online publiziert: 3. Februar 2021

(c) Springer-Verlag GmbH Deutschland, ein Teil

von Springer Nature 2021

\title{
Ausschreibung des Langener Wissenschaftspreises 2021
}

Der Langener Wissenschaftspreis wird für hervorragende wissenschaftliche Arbeiten auf den folgenden Gebieten ausgeschrieben:

- Infektiologie in der Human- und Veterinärmedizin (zum Beispiel Virologie, Bakteriologie, Immunologie),

- Hämatologie,

- Allergologie,

- Gen- und Zelltherapie und TissueEngineering,

- Erforschung von innovativen biomedizinischen Präventions- und Therapieansätzen,

- Arzneimittelsicherheit und Pharmako-Epidemiologie,

- damit in Verbindung stehende technologische Verfahren.

Der Langener Wissenschaftspreis ist mit $15.000 €$ dotiert und wird vom Verein zur Förderung des Langener Wissenschaftspreises e. V. gestiftet.

Nähere Informationen zu den Ausschreibungsbedingungen und eine Liste der einzureichenden Bewerbungsun- terlagen finden Sie unter www.pei.de/ langenerwissenschaftspreis bzw. unter www.langener-wissenschaftspreis.de.

Sowohl Einzel- als auch Gruppenbewerbungen sind möglich. Bewerbungen von jüngeren Wissenschaftlerinnen und Wissenschaftlern sind bevorzugt willkommen, auch wenn eine explizite Altersgrenze nicht festgelegt ist. Die Ausschreibung erfolgt deutschlandweit.

Die Bewerbungsunterlagen senden Sie bitte elektronisch an:

langener-wissenschaftspreis@pei.de

Die Bewerbungsfrist endet am 31.05.2021.

Unter den besten eingegangenen $\mathrm{Be}$ werbungen wird die Preisträgerin/der Preisträger im Rahmen einer wissenschaftlichen Vortragsveranstaltung im Sommer/Herbst am Paul-Ehrlich-Institut oder virtuell in einer Online-Veranstaltung ermittelt.

Die festliche Verleihung des Preises findet am 19.11.2021 voraussichtlich am Paul-Ehrlich-Institut statt. 